Journal of Animal and Veterinary Advances 11 (1): 67-73, 2012

ISSN: $1680-5593$

(C) Medwell Journals, 2012

\title{
Discovering the Hidden Biodiversity of Crustacea (Branchiopoda, Maxillopoda and Ostracoda) Assemblages in the High Mountain Lakes of Kackar Mountains (Turkey)
}

\author{
Cem Aygen, Didem Ozdemir Mis and M. Rusen Ustaoglu \\ Department of Marine-Inland Water Sciences and Technology, Faculty of Fisheries, \\ Ege University, 35100 Bornova, Izmir, Turkey
}

\begin{abstract}
The Branchiopoda, Maxillopoda and Ostracoda fauna of 59 high mountain lakes of Kackar mountains was investigated for the first time. During the study, six expeditions were carried out in the Summers of 2005-2007. All lakes are located at altitudes between 2530 and $3370 \mathrm{~m}$ except Uzungol (1100 m). Beside the biological samplings, major environmental variables of the lakes were measured. A total of 21 Branchiopoda, 9 Maxillopoda and 3 Ostracod taxa were identified. All the taxa identified are new records for their localities except C. ponticus. The investigated ecosystems support a high micro-crustacean diversity that must be adequately preserved due to the increasing direct and indirect impacts on high mountain lakes.
\end{abstract}

Key words: Branchiopoda, maxillopoda, ostracoda, Kackar mountains, biodivesity, Eastern Black sea, Turkey

\section{INTRODUCTION}

High mountain lakes are extreme ecosystems and at first glance, appear inappropriate environments. Nutrient deficiency, low temperatures intensive UV radiation or darkness for months make high mountain lakes extraordinary aquatic habitats.

Because high mountain lakes are small and their trophic webs are less complex than those of lowland lakes they are suitable for measuring and understanding ecological processes.

Often located in remote areas, high mountain lakes are also studied to investigate community structure and flow in isolation from human influence, subject only to natural forcing (Such as climate variability) or to long-range human impact such as the impact of atmospheric pollutants.

In the late 19th and early 20th centuries many high-altitude lakes in the European Alps were inventoried and their plankton fauna and flora were described in detail. There are number of recent studies on the micro-crustaceans of Turkey but almost all of these were carried out in lowland lakes. Only a few studies of high mountain lakes are available.

A recent study of the zooplankton species diversity is very high (Ustaoglu et al., 2005; Aygen et al., 2009). Additionally, some rare species such as Hemidiaptomus kummerloewei Mann, 1940 included in the IUCN red list of threatened species, only occurs in those sheltered areas. The lack of previous studies dealing with high mountain lakes limits the ability to adequately conserve these unique environments. This study therefore, presents results of a study on the composition of crustaceans in the high mountain lakes of Eastern Black sea coast of Turkey.

Study area: The Pontic mountain range (Eastern Black sea coast) is one of the three major glacier regions of Turkey. The highest peak of the Pontic range is Mount Kackar $(3932 \mathrm{~m})$ where five glaciers are formed. Some of the lakes studied located within the Kackar Mountains National park.

The National park is situated in the East Black sea region in the North-Eastern corner of Turkey. The park was created in 1994 and covers 51,500 ha.

Results of this study also will contribute to biodiversity of the Kackar Mountains National park. The locations of the 59 lakes studied as well as the characteristics of the sampling sites are shown in Fig. 1 and Table 1.

The lakes in Kackar mountains are small in size (From 0.03-8.9 ha) and shallow (Maximum depths ranging from $<1 \mathrm{~m}$ up to $49 \mathrm{~m}$ ). They remain frozen from October or November until May or June and their small size brings about rapid gains and loss in heat. When the altitudes of the lakes take into account, all lakes situated between 2530 and $3370 \mathrm{~m}$ except Uzungol (1100 m).

Corresponding Author: Cem Aygen, Department of Marine-Inland Water Sciences and Technology, Faculty of Fisheries, Ege University, 35100 Bornova, Izmir, Turkey 


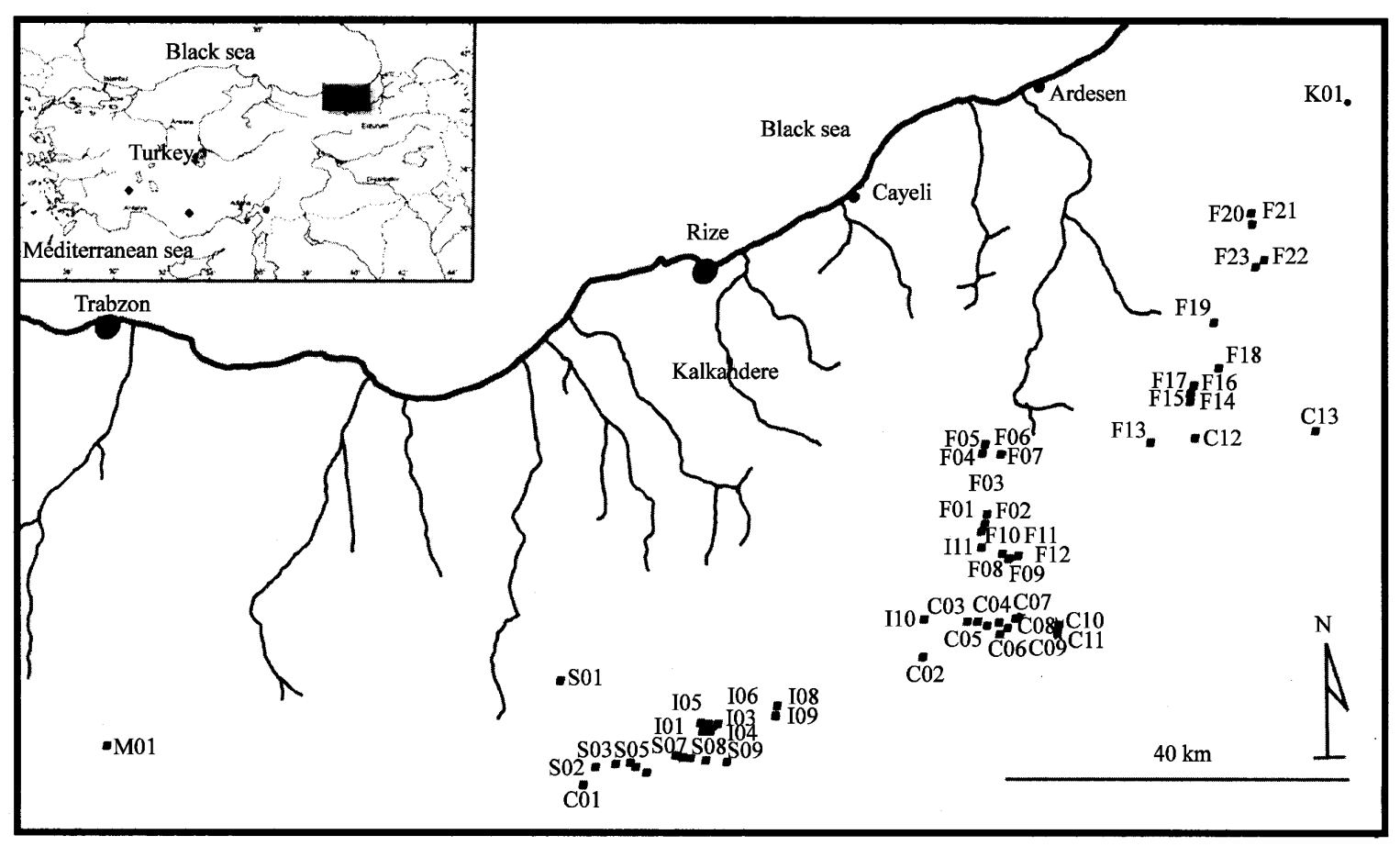

Fig. 1: Map of Eastern Black sea range in Turkey

\begin{tabular}{|c|c|c|c|c|c|}
\hline Basin code & Date & Locality & Basin & Altitude & Coordinates \\
\hline $\mathrm{C} 01$ & 30.07 .2007 & Goloba lake (Valley of Goloba) & Coruh river & 2540 & $\begin{array}{l}40^{\circ} 30^{\prime} 36^{\prime \prime} \mathrm{N} \\
40^{\circ} 19^{\prime} 12^{\prime \prime} \mathrm{E}\end{array}$ \\
\hline $\mathrm{C} 02$ & 19.08 .2005 & Dagbasi lake (Valley of Ovit) & Coruh river & 2710 & $\begin{array}{l}40^{\circ} 37^{\prime} 02^{\prime \prime} \mathrm{N} \\
40^{\circ} 46^{\prime} 47^{\prime \prime} \mathrm{E}\end{array}$ \\
\hline $\mathrm{C} 03$ & 04.08 .2006 & Batiaksu lake (Valley of Aksu) & Coruh river & 3050 & $\begin{array}{l}40^{\circ} 39^{\prime} 13^{\prime \prime} \mathrm{N} \\
40^{\circ} 50^{\prime} 39^{\prime \prime} \mathrm{E}\end{array}$ \\
\hline $\mathrm{C} 04$ & 04.08 .2006 & Kuzeyaksu lake (Valley of Aksu) & Coruh river & 3070 & $\begin{array}{l}40^{\circ} 39^{\prime} 19^{\prime \prime} \mathrm{N} \\
40^{\circ} 50^{\prime} 577^{\prime \prime} \mathrm{E}\end{array}$ \\
\hline $\mathrm{C} 05$ & 04.08 .2006 & Doguaksu lake (Valley of Aksu) & Coruh river & 3120 & $\begin{array}{l}40^{\circ} 39^{\prime} 09^{\prime \prime} \mathrm{N} \\
40^{\circ} 51^{\prime} 06^{\prime \prime} \mathrm{E}\end{array}$ \\
\hline $\mathrm{C} 06$ & 26.07.2007 & Ortagol lake (Ovit Yedigoller) & Coruh river & 2960 & $\begin{array}{l}40^{\circ} 38^{\prime} 51^{\prime \prime} \mathrm{N} \\
40^{\circ} 52^{\prime} 09^{\prime \prime} \mathrm{E}\end{array}$ \\
\hline $\mathrm{C} 07$ & 19.08 .2005 & Ustgol lake (Mor yayla Yedigoller) & Coruh river & 3030 & $\begin{array}{l}40^{\circ} 38^{\prime} 511^{\prime \prime N} \\
40^{\circ} 52^{\prime} 54^{\prime \prime} \mathrm{E}\end{array}$ \\
\hline $\mathrm{C} 08$ & $\begin{array}{l}06.07 .2005 \\
19.08 .2005\end{array}$ & Adaligol lake (Mor yayla Yedigoller) & Coruh river & 3020 & $\begin{array}{l}40^{\circ} 38^{\prime} 43^{\prime \prime} \mathrm{N} \\
40^{\circ} 53^{\prime} 10^{\prime \prime} \mathrm{E}\end{array}$ \\
\hline $\mathrm{C} 09$ & $\begin{array}{l}06.07 .2005 \\
19.08 .2005\end{array}$ & Ortagol lake (Mor yayla Yedigoller) & Coruh river & 3010 & $\begin{array}{l}40^{\circ} 38^{\prime} 53^{\prime \prime} \mathrm{N} \\
40^{\circ} 53^{\prime} 18^{\prime \prime} \mathrm{E}\end{array}$ \\
\hline $\mathrm{C} 10$ & $\begin{array}{l}06.07 .2005 \\
19.08 .2005\end{array}$ & Buyukgol lake (Mor yayla Yedigoller) & Coruh river & 2980 & $\begin{array}{l}40^{\circ} 38^{\prime} 45^{\prime \prime} \mathrm{N} \\
40^{\circ} 53^{\prime} 36^{\prime \prime} \mathrm{E}\end{array}$ \\
\hline C11 & 19.08 .2005 & Altgol lake (Mor yayla Yedigoller) & Coruh river & 2950 & $\begin{array}{l}40^{\circ} 38^{\prime} 53^{\prime \prime} \mathrm{N} \\
40^{\circ} 53^{\prime} 40^{\prime \prime} \mathrm{E}\end{array}$ \\
\hline $\mathrm{C} 12$ & 23.08 .2006 & Deniz lake (Valley of Bahral) & Coruh river & 3370 & $\begin{array}{l}40^{\circ} 49^{\prime} 07^{\prime \prime} \mathrm{N} \\
41^{\circ} 09^{\prime} 39^{\prime \prime} \mathrm{E}\end{array}$ \\
\hline $\mathrm{C} 13$ & 24.08 .2006 & Kartal lake (Valley of Bahral) & Coruh river & 2940 & $\begin{array}{l}40^{\circ} 50 \prime 20^{\prime \prime} \mathrm{N} \\
41^{\circ} 18^{\prime} 04^{\prime \prime} \mathrm{E}\end{array}$ \\
\hline $\mathrm{C} 14$ & 24.08 .2006 & Devise lake (Valley of Bahral) & Coruh river & 2935 & $\begin{array}{l}40^{\circ} 50^{\prime} 22^{\prime \prime} \mathrm{N} \\
41^{\circ} 18^{\prime} 12^{\prime \prime} \mathrm{E}\end{array}$ \\
\hline F01 & 22.08 .2005 & Keci lake (Valley of Cermes) & Firtina stream & 3070 & $\begin{array}{l}40^{\circ} 44^{\prime} 25^{\prime \prime} \mathrm{N} \\
40^{\circ} 51^{\prime} 50^{\prime \prime} \mathrm{E}\end{array}$ \\
\hline F02 & 22.08 .2005 & Cermes Karagol lake (Valley of Cermes) & Firtina stream & 2990 & $\begin{array}{l}40^{\circ} 44^{\prime} 37^{\prime \prime} \mathrm{N} \\
40^{\circ} 52^{\prime} 04^{\prime \prime} \mathrm{E}\end{array}$ \\
\hline F03 & $\begin{array}{l}09.07 .2005 \\
22.08 .2005\end{array}$ & Cermes lake (Valley of Cermes) & Firtina stream & 2780 & $\begin{array}{l}40^{\circ} 44^{\prime} 58^{\prime \prime} \mathrm{N} \\
40^{\circ} 52^{\prime} 09^{\prime \prime} \mathrm{E}\end{array}$ \\
\hline
\end{tabular}


J. Anim. Vet.Adv., 11 (1):67-73, 2012

Table 1: Continued

\begin{tabular}{|c|c|c|c|c|c|}
\hline Basin code & Date & Locality & Basin & Altitude & Coordinates \\
\hline F04 & 25.08 .2007 & Kayakaynak lake (Valley of Ambarlik) & Firtina stream & 3080 & $\begin{array}{l}40^{\circ} 49^{\prime} 17^{\prime \prime} \mathrm{N} \\
40^{\circ} 52^{\prime} 43^{\prime \prime} \mathrm{E}\end{array}$ \\
\hline F05 & 25.08 .2007 & Buyuk Balikli lake (Valley of Ambarlik) & Firtina stream & 2990 & $\begin{array}{l}40^{\circ} 49^{\prime} 28^{\prime \prime} \mathrm{N} \\
40^{\circ} 52^{\prime} 51^{\prime \prime} \mathrm{E}\end{array}$ \\
\hline F06 & 01.08 .2006 & Sirpal lake (Valley of Cahberik) & Firtina stream & 2940 & $\begin{array}{l}40^{\circ} 49^{\prime} 21^{\prime \prime} \mathrm{N} \\
40^{\circ} 53^{\prime} 40^{\prime \prime} \mathrm{E}\end{array}$ \\
\hline $\mathrm{F} 07$ & 01.08 .2006 & Cahberik lake (Valley of Cahberik) & Firtina stream & 2810 & $\begin{array}{l}40^{\circ} 49^{\prime} 17^{\prime} \mathrm{N} \\
40^{\circ} 54^{\prime} 09^{\prime \prime} \mathrm{E}\end{array}$ \\
\hline F08 & $\begin{array}{l}08.07 .2005 \\
21.08 .2005\end{array}$ & Atmeydani lake (Valley of Vercenik) & Firtina stream & 2910 & $\begin{array}{l}40^{\circ} 43^{\prime} 11^{\prime \prime N} \\
40^{\circ} 54^{\prime} 01^{\prime \prime} \mathrm{E}\end{array}$ \\
\hline F09 & $\begin{array}{l}08.07 .2005 \\
21.08 .2005\end{array}$ & Kumlugol lake (Valley of Vercenik) & Firtina stream & 2860 & $\begin{array}{l}40^{\circ} 43^{\prime} 22^{\prime \prime} \mathrm{N} \\
40^{\circ} 54^{\prime} 17^{\prime \prime} \mathrm{E}\end{array}$ \\
\hline F10 & $\begin{array}{l}08.07 .2005 \\
21.08 .2005\end{array}$ & Incegol lake (Valley of Vercenik) & Firtina stream & 2915 & $\begin{array}{l}40^{\circ} 43^{\prime} 06^{\prime \prime} \mathrm{N} \\
40^{\circ} 54^{\prime} 23^{\prime \prime} \mathrm{E}\end{array}$ \\
\hline F11 & 21.08 .2005 & Buyuk Kapili lake (Valley of Vercenik) & Firtina stream & 3000 & $\begin{array}{l}40^{\circ} 43^{\prime} 00^{\prime \prime N} \\
40^{\circ} 54^{\prime} 54^{\prime \prime} \mathrm{E}\end{array}$ \\
\hline $\mathrm{F} 12$ & 21.08 .2005 & Altkapili lake (Valley of Vercenik) & Firtina stream & 3000 & $\begin{array}{l}40^{\circ} 43^{\prime} 11^{\prime \prime N} \\
40^{\circ} 54^{\prime} 57^{\prime \prime} \mathrm{E}\end{array}$ \\
\hline F13 & 31.07 .2006 & Kiblekay a lake (Valley of Apivanak) & Firtina stream & 2870 & $\begin{array}{l}40^{\circ} 49^{\prime} 24^{\prime \prime N} \\
41^{\circ} 06^{\prime} 06^{\prime \prime} \mathrm{E}\end{array}$ \\
\hline F14 & $\begin{array}{l}11.07 .2005 \\
24.08 .2005\end{array}$ & Buyukdeniz lake (Valley of Kavron) & Firtina stream & 2900 & $\begin{array}{l}40^{\circ} 52^{\prime} 09^{\prime} \mathrm{N} \\
41^{\circ} 09^{\prime} 42^{\prime \prime} \mathrm{E}\end{array}$ \\
\hline F15 & 24.08 .2005 & Meterez lake (Valley of Kavron) & Firtina stream & 2990 & $\begin{array}{l}40^{\circ} 51^{\prime} 49^{\prime \prime} \mathrm{N} \\
41^{\circ} 09^{\prime} 45^{\prime \prime} \mathrm{E}\end{array}$ \\
\hline F16 & $\begin{array}{l}11.07 .2005 \\
24.08 .2005\end{array}$ & Isimsiz lake (Valley of Kavron) & Firtina stream & 2890 & $\begin{array}{l}40^{\circ} 52^{\prime} 28^{\prime \prime N} \\
41^{\circ} 09^{\prime} 46^{\prime \prime} \mathrm{E}\end{array}$ \\
\hline F17 & 24.08 .2005 & Karadeniz lake (Valley of Ceymakcur) & Firtina stream & 2770 & $\begin{array}{l}40^{\circ} 52^{\prime} 42^{\prime \prime N} \\
41^{\circ} 10^{\prime} 03^{\prime \prime} \mathrm{E}\end{array}$ \\
\hline F18 & 29.07 .2006 & Ceymakcur lake (Valley of Ceymakcur) & Firtina stream & 2650 & $\begin{array}{l}40^{\circ} 53^{\prime} 44^{\prime \prime} \mathrm{N} \\
41^{\circ} 11^{\prime} 30^{\prime \prime} \mathrm{E}\end{array}$ \\
\hline F19 & 30.07 .2006 & Buyukgol lake (Valley of Avusor) & Firtina stream & 2670 & $\begin{array}{l}40^{\circ} 56^{\prime} 13^{\prime \prime} \mathrm{N} \\
41^{\circ} 12^{\prime} 02^{\prime \prime} \mathrm{E}\end{array}$ \\
\hline $\mathrm{F} 20$ & 22.08 .2007 & Tobamizga lake (Valley of Tunca) & Firtina stream & 2620 & $\begin{array}{l}41^{\circ} 02^{\prime} 19^{\prime} \mathrm{N} \\
41^{\circ} 1537^{\prime \prime} \mathrm{E}\end{array}$ \\
\hline $\mathrm{F} 21$ & 22.08 .2007 & Kucuk Tobamizga lake (Valley of Tunca) & Firtina stream & 2630 & $\begin{array}{l}41^{\circ} 02^{\prime} 08^{\prime \prime} \mathrm{N} \\
41^{\circ} 15^{\prime} 39^{\prime \prime} \mathrm{E}\end{array}$ \\
\hline $\mathrm{F} 22$ & 23.08 .2007 & Buyuk Ciftegol lake (Valley of Tunca) & Firtina stream & 2600 & $\begin{array}{l}40^{\circ} 59^{\prime} 24^{\prime \prime N} \\
41^{\circ} 15^{\prime} 41^{\prime \prime} \mathrm{E}\end{array}$ \\
\hline F23 & 23.08 .2007 & Kucuk Ciftegol lake (Valley of Tunca) & Firtina stream & 2550 & $\begin{array}{l}40^{\circ} 59^{\prime} 36^{\prime \prime} \mathrm{N} \\
41^{\circ} 15^{\prime} 49^{\prime \prime} \mathrm{E}\end{array}$ \\
\hline I01 & 20.08 .2006 & Dipsiz lake (Valley of Anzer) & Iyidere & 2670 & $\begin{array}{l}40^{\circ} 33^{\prime} 28^{\prime} \mathrm{N} \\
40^{\circ} 28^{\prime} 25^{\prime \prime} \mathrm{E}\end{array}$ \\
\hline $\mathrm{I} 02$ & 25.07 .2007 & Koyun lake (Valley of Anzer) & Iyidere & 3010 & $\begin{array}{l}40^{\circ} 31^{\prime} 34^{\prime \prime} \mathrm{N} \\
40^{\circ} 28^{\prime} 58^{\prime \prime} \mathrm{E}\end{array}$ \\
\hline I03 & 20.08 .2006 & Kucukhatalan lake (Valley of Anzer) & Iyidere & 2800 & $\begin{array}{l}40^{\circ} 33^{\prime} 16^{\prime \prime N} \\
40^{\circ} 29^{\prime} 22^{\prime \prime} \mathrm{E}\end{array}$ \\
\hline I04 & 20.08 .2006 & Hatalan lake (Valley of Anzer) & Iyidere & 2810 & $\begin{array}{l}40^{\circ} 33^{\prime} 11^{\prime \prime} \mathrm{N} \\
40^{\circ} 29^{\prime} 24^{\prime \prime} \mathrm{E}\end{array}$ \\
\hline I05 & 20.08 .2006 & Kucuksivri lake (Valley of Anzer) & Iyidere & 2710 & $\begin{array}{l}40^{\circ} 33^{\prime} 36^{\prime \prime} \mathrm{N} \\
40^{\circ} 29^{\prime} 50^{\prime \prime} \mathrm{E}\end{array}$ \\
\hline I06 & 20.08 .2006 & Sivrinin lake (Valley of Anzer) & Iyidere & 2700 & $\begin{array}{l}40^{\circ} 33^{\prime} 39^{\prime} \mathrm{N} \\
40^{\circ} 29^{\prime} 52^{\prime \prime} \mathrm{E}\end{array}$ \\
\hline I07 & 02.08 .2006 & Akcaagil lake (Valley of Anzer) & Iyidere & 2940 & $\begin{array}{l}40^{\circ} 31^{\prime} 19^{\prime \prime} \mathrm{N} \\
40^{\circ} 30^{\prime} 40^{\prime \prime} \mathrm{E}\end{array}$ \\
\hline I08 & 21.08 .2006 & Katrec lake (Valley of Arzayan) & Iyidere & 2700 & $\begin{array}{l}40^{\circ} 34^{\prime} 06^{\prime \prime} \mathrm{N} \\
40^{\circ} 34^{\prime} 51^{\prime \prime} \mathrm{E}\end{array}$ \\
\hline I09 & 21.08 .2006 & Kucukkatrec Lake (Valley of Arzayan) & Iyidere & 2690 & $\begin{array}{l}40^{\circ} 34^{\prime} 13^{\prime \prime} \mathrm{N} \\
40^{\circ} 34^{\prime} 58^{\prime \prime} \mathrm{E}\end{array}$ \\
\hline $\mathrm{I} 10$ & 03.08 .2006 & Citrik lake (Valley of Cimil) & Iyidere & 2850 & $\begin{array}{l}40^{\circ} 39^{\prime} 31^{\prime \prime} \mathrm{N} \\
40^{\circ} 46^{\prime} 59^{\prime \prime} \mathrm{E}\end{array}$ \\
\hline I11 & 03.08 .2006 & Salar lake (Valley of Cimil) & Iyidere & 2820 & $\begin{array}{l}40^{\circ} 43 ' 28^{\prime \prime} \mathrm{N} \\
40^{\circ} 52^{\prime} 09^{\prime \prime} \mathrm{E}\end{array}$ \\
\hline K01 & 25.08 .2006 & Arhavi Karagol lake (Valley of Kabisra) & Kabisra stream & 2660 & $\begin{array}{l}41^{\circ} 09^{\prime} 28^{\prime \prime N} \\
41^{\circ} 24^{\prime} 19^{\prime \prime} \mathrm{E}\end{array}$ \\
\hline M01 & 26.08 .2007 & Cakir Lake (Valley of Altindere) & Macka stream & 2530 & $\begin{array}{l}40^{\circ} 34^{\prime} 34^{\prime \prime N} \\
39^{\circ} 41^{\prime} 26^{\prime \prime} \mathrm{E}\end{array}$ \\
\hline S01 & 26.08 .2006 & Uzungol lake (Valley of Uzungol) & Solakli stream & 1100 & $\begin{array}{l}40^{\circ} 37^{\prime} 14^{\prime \prime N} \\
40^{\circ} 17^{\prime} 44^{\prime \prime} \mathrm{E} \\
\end{array}$ \\
\hline
\end{tabular}




\begin{tabular}{|c|c|c|c|c|c|}
\hline Basin code & Date & Locality & Basin & Altitude & Coordinates \\
\hline $\mathrm{S} 02$ & 30.07 .2007 & Kirklarcami lake (Valley of Uzungol) & Solakli stream & 2740 & $\begin{array}{l}40^{\circ} 31^{\prime} 46^{\prime \prime} \mathrm{N} \\
40^{\circ} 20^{\prime} 06^{\prime \prime} \mathrm{E}\end{array}$ \\
\hline $\mathrm{S} 03$ & 29.07 .2007 & Multat Karagol lake (Valley of Uzungol) & Solakli stream & 2800 & $\begin{array}{l}40^{\circ} 31^{\prime} 30^{\prime \prime} \mathrm{N} \\
40^{\circ} 21^{\prime} 46^{\prime \prime} \mathrm{E}\end{array}$ \\
\hline S04 & 27.07 .2007 & Balik lake (Valley of Uzungol) & Solakli stream & 2570 & $\begin{array}{l}40^{\circ} 31^{\prime} 54^{\prime \prime} \mathrm{N} \\
40^{\circ} 23^{\prime} 01^{\prime \prime} \mathrm{E}\end{array}$ \\
\hline S05 & 27.07 .2007 & Aygir lake (Valley of Uzungol) & Solakli stream & 2710 & $\begin{array}{l}40^{\circ} 31^{\prime} 39^{\prime \prime} \mathrm{N} \\
40^{\circ} 23^{\prime} 28^{\prime \prime} \mathrm{E}\end{array}$ \\
\hline S06 & 29.07 .2007 & Saricicek lake (Valley of Uzungol) & Solakli stream & 2880 & $\begin{array}{l}40^{\circ} 31^{\prime} 15^{\prime \prime} \mathrm{N} \\
40^{\circ} 24^{\prime} 21^{\prime \prime} \mathrm{E}\end{array}$ \\
\hline S07 & 28.07 .2007 & Demirkapi Karagol lake (Valley of Uzungol) & Solakli stream & 2930 & $\begin{array}{l}40^{\circ} 31^{\prime} 41^{\prime \prime N} \\
40^{\circ} 27^{\prime} 03^{\prime \prime} \mathrm{E}\end{array}$ \\
\hline $\mathrm{S} 08$ & 28.07 .2007 & Piromer lake (Valley of Uzungol) & Solakli stream & 2870 & $\begin{array}{l}40^{\circ} 32^{\prime} 00^{\prime \prime} \mathrm{N} \\
40^{\circ} 27^{\prime} 09^{\prime \prime} \mathrm{E}\end{array}$ \\
\hline S09 & 28.07 .2007 & Buz lake (Valley of Uzungol) & Solakli stream & 3040 & $\begin{array}{l}40^{\circ} 31^{\prime} 58^{\prime \prime} \mathrm{N} \\
40^{\circ} 27^{\prime} 36^{\prime \prime} \mathrm{E}\end{array}$ \\
\hline
\end{tabular}

\section{MATERIALS AND METHODS}

In order to study the micro-crustacean fauna of 59 mountain lakes in the Eastern Black sea range, six excursions were conducted in 2005-2007. Due to the high altitude most of the sites studied are generally covered by snow and ice during 8-9 months of the year and ice-free periods occurs only in warm months i.e., June-August. Qualitative samples were collected by horizontally and vertical hauls, using standart plankton net (Mesh size $60 \mu \mathrm{m})$. For the quantitative analyses, a $10 \mathrm{~L}$. sampling container was filled and the samples pass through a $50 \mu \mathrm{m}$ mesh. The zooplankton were washed from the filter into a jar. Also, samples taken from the littoral zone were collected with a plankton hand net (Mesh size $250 \mu \mathrm{m}$ ). All samples were kept in $330 \mathrm{~mL}$ PVC jars and fixed in $4 \%$ formaldehyde. Water samples were taken from the littoral of each lake. Six environmental variables, namely water temperature (Temp., ${ }^{\circ} \mathrm{C}$ ), dissolved oxygen $\left(\mathrm{DO}, \mathrm{mg} \mathrm{L}^{-1}\right), \mathrm{pH}$, Electrical Conductivity $\left(\mathrm{EC}, \mu \mathrm{s} \mathrm{cm}^{-1}\right.$ ) and Secchi depths were measured in situ by using a WTW pH-meter (model 330), a WTW oxygen-meter (model 330) and an YSI 30 model SCT-meter and a secchi disc. Other variables $\left(\mathrm{NO}_{2}{ }^{-}-\mathrm{N}, \mathrm{NO}_{3}{ }^{-}-\mathrm{N}, \mathrm{NH}_{4}^{-}-\mathrm{N}, \mathrm{PO}_{4}{ }^{-3}-\mathrm{P}\right.$, $\mathrm{HCO}_{3}^{-}, \mathrm{Ca}^{+2}, \mathrm{Mg}^{+2}, \mathrm{SiO}_{2}{ }^{-}, \mathrm{Chl}$.-a, etc.) were measured in the laboratory after following the standard methods of APHA (1989). For taxonomical identification of the specimens, the keys by Smirnov $(1992,1996)$, Benzie (2005), Korovchinski (1992), Beladjal and Mertens (1997), Reddy (1994), Bronstein (1947), Hendreson (1990) and Meisch (2000) were used.

\section{RESULTS AND DISCUSSION}

Environmental variables: Minimum and maximum values of the environmental variables of studied lakes are shown in the Table 2. In terms of water quality, all of the lakes can be classified as 1st class because in terms of their
Table 2: Minimum and maximum values of the environmental variables

\begin{tabular}{|c|c|c|}
\hline Variables & Min. & Max. \\
\hline Secchi depth $(\mathrm{cm})$ & 50.00 & 1100.00 \\
\hline Temperature $\left({ }^{\circ} \mathrm{C}\right)$ & 2.50 & 21.00 \\
\hline $\mathrm{pH}$ & 6.75 & 9.71 \\
\hline $\mathrm{DO}\left(\mathrm{mg} \mathrm{L}^{-1}\right)$ & 5.30 & 10.10 \\
\hline $\mathrm{EC}\left(\mu \mathrm{S} 25^{\circ} \mathrm{C} \mathrm{cm}^{-1}\right)$ & 12.00 & 121.80 \\
\hline$d^{\circ} \mathrm{H}$ & 0.80 & 3.40 \\
\hline $\mathrm{HCO}_{3}^{-}\left(\mathrm{mg} \mathrm{L}^{-1}\right)$ & 18.30 & 73.20 \\
\hline $\mathrm{Ca}^{+2}\left(\mathrm{mg} \mathrm{L}^{-1}\right)$ & 8.00 & 32.10 \\
\hline $\mathrm{Mg}^{+2}\left(\mathrm{mg} \mathrm{L}^{-1}\right)$ & 4.86 & 19.46 \\
\hline $\mathrm{NO}_{2}^{-}-\mathrm{N}\left(\mu \mathrm{g} \mathrm{L}^{-1}\right)$ & 0.00 & 5.21 \\
\hline $\mathrm{NO}_{3}{ }^{-}-\mathrm{N}\left(\mu \mathrm{g} \mathrm{L}^{-1}\right)$ & 0.00 & 105.00 \\
\hline $\mathrm{NH}_{4}{ }^{-}-\mathrm{N}\left(\mu \mathrm{g} \mathrm{L}^{-1}\right)$ & 0.00 & 106.90 \\
\hline $\mathrm{PO}_{4}^{-3}-\mathrm{P}\left(\mu \mathrm{g} \mathrm{L}^{-1}\right)$ & 0.00 & 10.95 \\
\hline $\mathrm{SiO}_{2}^{-}\left(\mu \mathrm{g} \mathrm{L}^{-1}\right)$ & 258.50 & 2516.30 \\
\hline Chlorophyll-a $\left(\mu \mathrm{g} \mathrm{L}^{-1}\right)$ & 0.00 & 2.32 \\
\hline
\end{tabular}

ammonium, nitrite, nitrate nitrogen levels and total phosphorus level. Similarly, the quality classes of the lakes were changed between I-III according to levels of dissolved oxygen. When the Total Nitrogen (TN) and Total Phosphourus (TP) are taking into consideration, all the lakes show oligotrophic character. Chlorophyll-a concentration of the lakes were generally low and this character also support the result is given above.

Environmental variables data was used to show relationships among the lakes by means of using test of Unweighted Pair Group Mean Averages (UPGMA) analysis. In this analysis, logarithmic transformation was applied to all data except $\mathrm{pH}$. Result of UPGMA clustering showed that the similarity of the lakes is $>88 \%$ (Fig. 2 ).

Taxonomic structure: In total, 33 Crustacean taxa, comprising 21 branchiopods, 9 copepods and 3 ostracods, respectively were identified (Table 3). All the taxa identified are new records for their localities except C. ponticus.

Among the copepods, A. acutilobatus was the dominant species (In 5 basins at 46 stations). $A$. acutilobatus is important copepod species and reaches high densities at the water bodies of mountainous regions 


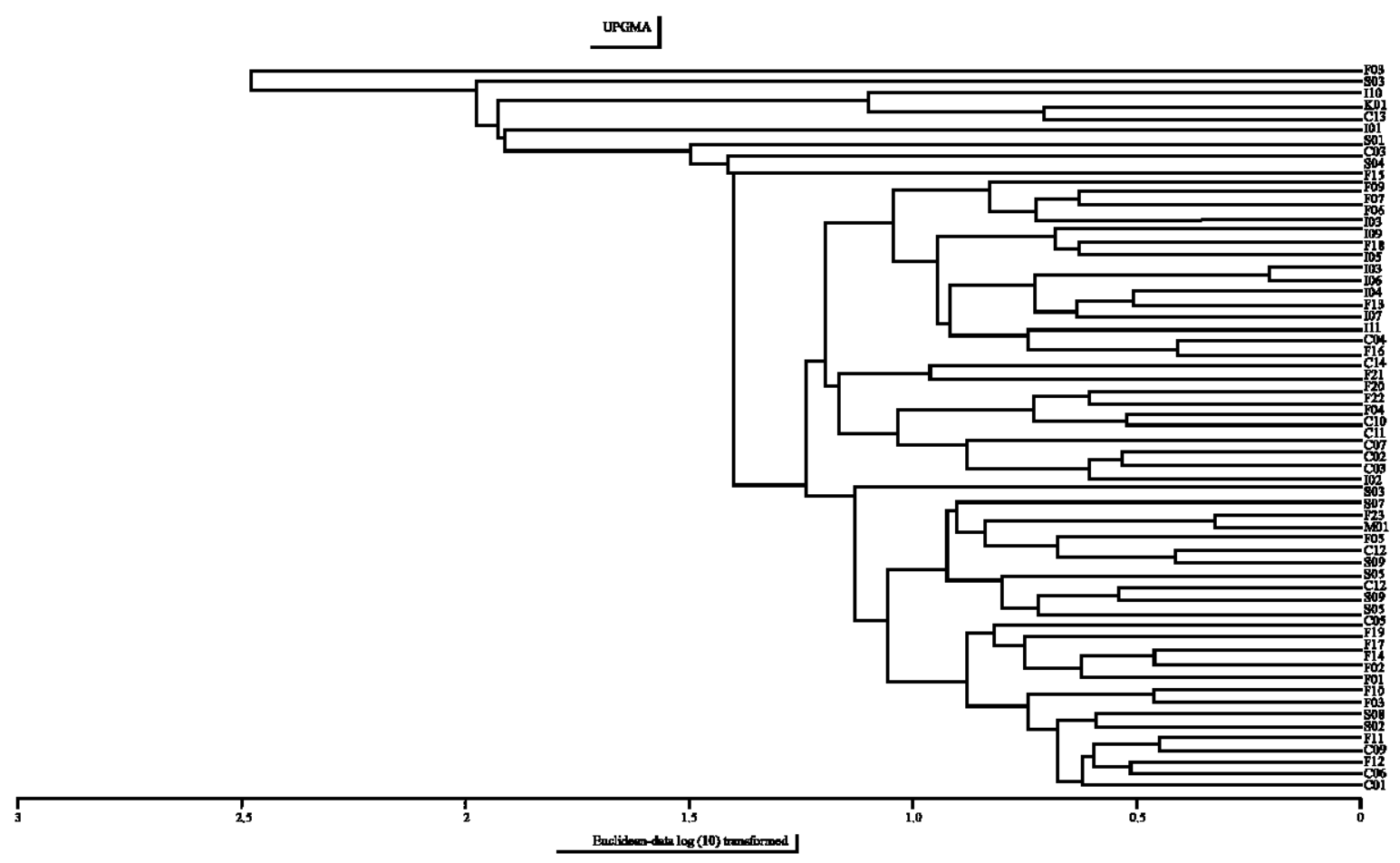

Fig. 2: Result of UPGMA clustering showed that the similarity of the lakes

Table 3: Branchiopoda, Maxillopoda and Ostracoda composition and distribution of the lakes

\begin{tabular}{|c|c|c|c|c|c|c|}
\hline \multirow[b]{2}{*}{ Species of crustacean } & \multicolumn{6}{|l|}{ Basin } \\
\hline & Coruh & Firtina & Iyidere & Kabisra & Macka & Solakli \\
\hline \multicolumn{7}{|l|}{ Branchiopoda } \\
\hline Diophanosoma brachyurum (Lievin,1848) & $\mathrm{C} 8$ & - & - & - & - & - \\
\hline Daphnia cucullata (Sars, 1862) & - & F9 & - & - & - & - \\
\hline Daphnia curvicornis (Eylmann, 1887) & $\mathrm{C} 1, \mathrm{C} 12$ & F12 & $\mathrm{I} 3, \mathrm{I} 4$ & - & - & s9 \\
\hline Daphnia hyaline (Leydig, 1860) & - & $\mathrm{F} 20$ & - & - & M1 & - \\
\hline Daphnia longispina (O.F. Muller, 1875) & C6 & - & I6 & K1 & - & - \\
\hline Daphnia pulex (Leydig, 1860) & $\mathrm{C} 8, \mathrm{C} 9$ & F4, F11 & - & - & - & - \\
\hline Simocephalus exspinosus (Koch, 1841) & - & F8 & - & - & - & - \\
\hline Simocephalus vetulus (O.F. Muller, 1776) & $\mathrm{C} 3$ & - & 11 & - & - & - \\
\hline Ceriodophnia laticaudata (P.E. Muller, 1867) & $\mathrm{C} 8, \mathrm{C} 14$ & - & I1 & K1 & - & - $\quad$ \\
\hline Ceriodophnia quadrangula (O.F. Muller, 1785) & $\begin{array}{l}\mathrm{C} 1-\mathrm{C} 4, \mathrm{C} 7, \mathrm{C} 8 \\
\mathrm{C} 11, \mathrm{C} 13\end{array}$ & $\begin{array}{l}\text { F3, F5, F8, } \\
\text { F12, F16 }\end{array}$ & I10, I11 & - & - & $\mathrm{S} 1, \mathrm{~S} 4, \mathrm{~S} 5$ \\
\hline Scopholeberis kingi (Sars, 1903) & $\mathrm{C} 2$ & - & - & - & - & - \\
\hline Scapholeberis mucronata (O.F. Muller, 1785) & $\mathrm{C} 11$ & F8 & - & - & - & - \\
\hline Mocrothrix hirsuticornis (Norman and Brady, 1867) & $\mathrm{C} 5$ & $\begin{array}{l}\text { F7-F10, F12-F15, } \\
\text { F21, F22 }\end{array}$ & I3-I5, I8, I11 & - & - & S5 \\
\hline Bosmina longirostris (O.F. Muller, 1785) & $\mathrm{C} 2, \mathrm{C} 5, \mathrm{C} 7-\mathrm{C} 10$ & F2, F3, F8-F12 & I10 & - & - & S4 \\
\hline Alonella excisa (Fischer, 1854) & $\begin{array}{l}\mathrm{C} 3, \mathrm{C} 6, \mathrm{C} 9, \mathrm{C} 11, \\
\mathrm{C} 13, \mathrm{C} 14\end{array}$ & F8 & I11 & - & M1 & - \\
\hline Chydorus sphaericus (O.F. Muller, 1776) & $\begin{array}{l}\mathrm{C} 1-\mathrm{C} 6, \mathrm{C} 8, \mathrm{C} 10, \\
\mathrm{C} 11, \mathrm{C} 13, \mathrm{C} 14\end{array}$ & $\begin{array}{l}\text { F1-F4, F6, F8-F11, } \\
\text { F13-F17, F19-F23 }\end{array}$ & I1-I9, I11 & $\mathrm{K} 1$ & M1 & S3-S9 \\
\hline $\begin{array}{l}\text { Alona quadrangularis (O.F. Muller, 1785) } \\
\text { Alona rectangular (Sars, 1862) }\end{array}$ & $\mathrm{C} 4, \mathrm{C} 11$ & $\begin{array}{l}\text { F8, F10, F14 } \\
\text { F4, F21 }\end{array}$ & $\mathrm{I} 3, \mathrm{I} 4, \mathrm{I} 10, \mathrm{I} 11$ & - & M1 & - \\
\hline Biopertura affinis (Ley dig, 1860) & $\begin{array}{l}\text { C1-C3, } \\
\text { C8-C11, C14 }\end{array}$ & $\begin{array}{l}\text { F3, F5, F6-F10, } \\
\text { F14, F20, F21 }\end{array}$ & $\begin{array}{l}\text { I1, I3-I6, } \\
\text { I10, I11 }\end{array}$ & $\mathrm{K} 1$ & M1 & S1, S6 \\
\hline Cercopagis pengoi (Ostroumov, 1892) & $\mathrm{C} 8, \mathrm{C} 10$ & F11, F14 & - & - & - & - \\
\hline $\begin{array}{l}\text { Chirocephalus ponticus (Beladjal and Mertens, 1997) } \\
\text { Maxillopoda }\end{array}$ & - & F16, F17 & - & - & - & - \\
\hline Occidodiaptomus dischensis (Brehm, 1938) & C7 & F12 & - & - & - & - \\
\hline Arctodiaptomus acutilobatus (G.O. Sars, 1903) & $\mathrm{C} 1-\mathrm{C} 3, \mathrm{C} 5-\mathrm{C} 14$ & $\begin{array}{l}\text { F1-F5, F7, F8, } \\
\text { F10-F17, F19, } \\
\text { F20, F22, F23 }\end{array}$ & $\begin{array}{l}\text { I1-I4, I6, } \\
\text { I8, I10, I11 }\end{array}$ & $\mathrm{K} 1$ & - & $\begin{array}{l}\text { S2, S3, } \\
\text { S5-S9 }\end{array}$ \\
\hline
\end{tabular}




\begin{tabular}{|c|c|c|c|c|c|c|}
\hline \multirow[b]{2}{*}{ Species of crustacean } & \multicolumn{6}{|l|}{ Basin } \\
\hline & Coruh & Firtina & Iyidere & Kabisra & Macka & Solakli \\
\hline Eucyclops serrulatus (Fischer, 1851) & $\mathrm{C} 2, \mathrm{C} 8, \mathrm{C} 14$ & F3, F8, F9, F15 & $\mathrm{I} 3, \mathrm{I} 4$ & - & - & S1, S3 \\
\hline Cyclops abyssorum (G.O. Sars, 1863) & - & - & - & - & M1 & - \\
\hline Paracyclops fimbriatus (Fischer, 1853) & - & F10 & - & - & - & S1 \\
\hline Canthocamptus stophylinus (Jurine, 1820) & $\mathrm{C} 8, \mathrm{C} 10$ & - & - & - & - & S7 \\
\hline Bryocamptus zschokkei (Schmeil, 1893) & $\mathrm{C} 10$ & - & - & - & - & - \\
\hline Bryocamptus minutus (Claus, 1863) & $\mathrm{C} 10$ & - & - & - & - & - \\
\hline Attheyella crassa (G.O. Sars, 1863) & - & F8, F14, F16 & - & - & - & S7 \\
\hline \multicolumn{7}{|l|}{ Ostracoda } \\
\hline Prionocypris zenkeri (Chyzer and Toth, 1858) & $\mathrm{C} 10$ & - & - $\quad$ & - & - & - \\
\hline Candona neglecta (G.O. Sars, 1887) & $\mathrm{C} 5$ & F3, F10, F13-F16 & I3, I9-I11 & K1 & - & S1 \\
\hline Fabaeformiscandona balatonica (Daday, 1894) & - & - & - & - & - & S2 \\
\hline
\end{tabular}

of Caucasus (Reddy, 1994). This species was previously recorded from the different parts of Turkey such as Devegecidi dam lake (Gunduz, 1998), Hirfanli dam lake (Yigit and Altindag, 2005), Asi river Kesikkopru dam lake (Yigit, 2006), Yarseli dam lake, Sariyar dam lake (Atici et al., 2008). E. serrulatus (In 4 basins at 11 stations) is a cosmopolitan species and the 2nd most common copepod of studied lakes. According to Ustaoglu et al. (2005) this species is one of the most common copepods of the high mountain lakes located at Taurus mountains. One of the interesting findings of this study is encountering of $O$. dischensis in two lakes (Lake Ustgol and Altkapili). According to Kiefer, O. dischensis is endemic to high mountain lakes of Eastern Anatolia and no further records of the species until now. Distribution area of $O$. dischensis was expanded to North by this study. C. abyssorum, $P$. fimbriatus, C. staphylinus, $B$. zschokkei, B. minutusand and A. crassa were less frequently encountered species among the lakes of Kackar mountain range.

Among the branchiopods, C. sphaericus (48 lakes) and B. affinis (29 lakes) were the dominant species, respectively. Endemic $C$. ponticus was unique anostracan species sampled during the study and recorded from type locality. One of the unusual findings of the study is recording of predatory $C$. pengoi from four locality (Lake Buyuk Kapili, Buyukdeniz, Adaligol, Buyukgol). C. pengoi is euryhaline and eurytherm ponto-caspic species.

It prefers the brackish-water environment but has also been found in freshwater conditions and warm water environment (Gorokhova et al., 2000). This alien species previously recorded from Lake Terkos in Turkish Thrace (Guher, 2004) but never recorded from high mountain lakes. With this study, distribution area of the species in Turkey is expanded.

Three ostracod taxa were collected during the sampling periods (Table 3 ). C. neglecta was the most frequent species, collected from 12 sampling sites. The least frequent (Collected from only one lake) were $P$. zenkeri and $F$. balatonica. Regarding the species diversity of the stations, Station 22 (Lake Atmeydani) had the higher species richness (12 species) followed by Stations 8 and 10 (Lake Adaligol and Buyukgol, respectively) each with 11 species.

\section{CONCLUSION}

In this study from the point of view of the regional limnology, the high mountain lakes of the Kackar mountains appear to be homogeneous water bodies easily characterizable and with low levels of pollution. However, the high diversity recorded in the investigated lakes underlines the importance of these fragile habitats which are rarely considered in traditional ecological research. High mountain lakes are increasingly threatened by direct and indirect impacts such as changes in hydrological cycles, water pollution and physical alteration of the habitat structure. The reduction of anthropic impacts on these ecosystems will hopefully become a priority in conservation and restoration projects, especially in protected areas. The present survey will be useful as a baseline for future studies and contributions to the knowledge of Turkey's biodiversity.

\section{ACKNOWLEDGEMENTS}

This study was financially supported by The Scientific and Technical Research Council of Turkey (TUBITAK Project: 104/Y/183).

\section{REFERENCES}

APHA, 1989. Standard Methods for the Examination of Water and Waste Water. 17th Edn., American Public Health Association, Washington, DC. 
Atici, T., S. Ahiska, A. Altinda and D. Aydin, 2008. Ecological effects of some heavy metals $(\mathrm{Cd}, \mathrm{Pb}, \mathrm{Hg}$, $\mathrm{Cr})$ pollution of phytoplanktonic algae and zooplanktonic organisms in Sariyar Dam Reservoir in Turkey. Afr. J. Biotechnol., 7: 1972-1977.

Aygen, C., D. O. Mis, M.R. Ustaoglu and S. Balik, 2009. Zooplankton composition and abundance in Lake Egrigol, a High Mountain Lake (Gundogmus, Antalya). Turk. J. Zool., 33: 83-88.

Beladjal, L. and J. Mertens, 1997. Chirocephalus ponticus n.sp. (Crustacea: Anostraca) and its affinities to the other Turkish species of the genus. Hydrobiologia, 359: 101-111.

Benzie, J.A.H., 2005. The Genus Daphnia (Including Daphniopsis): Anomopoda: Daphniidae (Guides to the Identification of the Microinvertebrates of the Continental Waters of the World). Kenobi Productions, Leiden, ISBN: 978-9057821516, Pages: 376.

Bronstein, Z.S., 1947. Fresh-Water Ostracoda. Fauna of the USSR Crustaceans. Vol. 2, Academy of Sciences of the USSR Publishers, Moscow, Pages: 470.

Gorokhova, E., N. Aladin and H.J. Dumont, 2000. Further expansion of the genus Cercopagis (Crustacea, Branchiopoda, Onychopoda) in the Baltic Sea, with notes on the taxa present and their ecology. Hydrobiologia, 429: 207-218.

Guher, H., 2004. A study on morphological characters, spatial and seasonal densities and co-existence of two predatory Cladocera, Cercopagis pengoi (Ostroumov, 1891) and Cornigerius maeoticus (Pengo, 1879) in Lake Terkos, Turkey. Crustaceana, 77: 669-681.

Gunduz, E., 1998.. Sinodiaptomus sarsi (Rylov, 1923) (Copepoda, Calanoida) in Turkey. Hydrobiologia, 380: 9-13.
Hendreson, P.A., 1990. Freshwater Ostracods. In: Synopses of the British Fauna (New Series), Barnes, R.S.K. and J.H. Crothers (Eds.). The Linnean Society of London, Shrewsbury.

Korovchinski, N.M., 1992. Sididae and Holopedidae (Crustacea: Daphniiformes). Guides to the Identification of the Microinvertebrates of the World. Vol. 3, SPB Academic Publishing, The Hague, Pages: 82.

Meisch, C., 2000. Freshwater Ostracoda of Western and Central Europe. Spektrum Akademischer Verlag, Heidelberg, Berlin, Pages: 522.

Reddy, Y.R., 1994. Copepoda: Calanoida: Diaptomidae. Guides to the Identification of the Microinvertebrates of the Continental Waters of the World. SPB Academic Publishing, The Hague, Pages: 221.

Smirnov, N.N., 1992. The Macrothricidae of the World. Guides to the Identification of the Microinvertebrates of the Continental Waters of the World. SPB Academic Publishing, The Hague, Pages: 143.

Smirnov, N.N., 1996. Cladocera: The Chydorinae and Sayciinae (Chydoridae) of the World. Guides to the Identification of the Microinvertebrates of the Continental Waters of the World. SPB Academic Publishing, The Hague, Pages: 195.

Ustaoglu, M.R., S. Balik, D.M. Ozdemir and C. Aygen, 2005. The zooplankton of some mountain lakes in the Taurus range (Turkey). Zool. Middle East, 34: 101-108.

Yigit, S. and A. Altindag, 2005. A taxonomical study on the zooplankton fauna of Hirfanli Dam Lake (Kirsehir), Turkey. G.U. J. Sci., 18: 563-567.

Yigit, S., 2006. Analysis of the zooplankton community by the shannon-weaver index in Kesikkopru Dam Lake, Turkey. Ankara Universitesi Ziraat Fakultesi, Tarim Bilimleri Dergisi, 12: 216-220. 